\title{
Objetos de Aprendizagem: Uma comparação entre SCORM e IMS Learning Design
}

\author{
Renato Luís de Souza Dutra* \\ Liane Margarida Rockenbach Tarouco** \\ Centro Interdisciplinar de Novas Tecnologias da Educação \\ Universidade Federal do Rio Grande do Sul
}

\section{Introdução}

Diversas Tecnologias da Informação e Comunicação (TICs) têm sido discutidas com vistas à sua utilização na Educação. $O$ professor, antes com poucos recursos tecnológicos à sua disposição, hoje dispõe de um amplo leque de possibilidades e cabe a ele decidir a melhor maneira de aplicá-las.

Neste sentido a tecnologia de Objetos de Aprendizagem com suas diversas utilizações têm se mostrado uma alternativa bastante interessante. Modelos e padrões como o SCORM e Learning Design entre outros, têm cada vez mais angariado adeptos ao redor do mundo. Apesar de terem os Objetos de Aprendizagem como seu foco principal, os dois modelos se diferem bastante em sua estrutura. Enquanto o SCORM foca mais na interação individual entre o aluno e o conteúdo instrucional, o IMS Learning Design se volta para toda a atividade de aprendizagem.

O objetivo deste artigo é analisar os dois modelos sob o ponto de vista de ferramentas de auxílio ao professor no processo de ensino-aprendizagem.

\section{Objetos de Aprendizagem}

Há algum tempo os Objetos de Aprendizagem têm sido utilizados por diversos professores como recurso educacional, tanto para a educação à distância como para a educação presencial. O conceito de Objetos de Aprendizagem pode ser sumarizado como a idéia de recursos suplementares ao processo de aprendizagem, que possam ser reutilizados para apoiar a aprendizagem (TAROUCO, FABRE E TAMUSIUNAS, 2003). Hodgins (2001) argumenta que os Objetos de Aprendizagem sejam considerados como blocos de LEGO $^{\circledR}$ de conteúdos, ou seja, o conteúdo em uma forma mínima que possa ser montado em conjunto com outros conteúdos, construindo um contexto de aprendizagem.

Desta forma, argumenta Wiley (2001), seria possível aos projetistas dos cursos desenvolverem pequenos módulos ou componentes instrucionais que pudessem ser reutilizados diversas vezes em contextos diferentes.

Com a popularização da tecnologia de objetos de aprendizagem no apoio às atividades de ensino-aprendizagem, surgiram algumas iniciativas visando padronizar a especificação, a construção e a identificação dos objetos de aprendizagem. A adoção de

\footnotetext{
"Doutorando em Informática na Educação e Mestre em Ciência da Computação pela UFRGS

"Doutora em Engenharia Elétrica pela USP e Mestre em Ciência da Computação pela UFRGS 
modelos e padrões para o desenvolvimento de Objetos de Aprendizagem é cada vez mais importante, visto que o rápido avanço da tecnologia leva à possível substituição dos Ambientes Informatizados de Aprendizagem $\left(\mathrm{LMS}^{1}\right)$ com maior rapidez do que a obsolescência de um objeto de aprendizagem.

Dentre os modelos existentes, o SCORM com uma abordagem mais centrada na autonomia e auto-aprendizagem é atualmente o mais popular e com o maior número de ferramentas de suporte.

\subsection{SCORM}

O Sharable Content Object Reference Model (SCORM) é um modelo de referência, ou seja, conjunto unificado de especificações para a disponibilização de conteúdos e serviços de e-learning. Este conjunto de especificações define um modelo de agregação de conteúdo, um modelo de seqüenciamento e um ambiente de execução para objetos de aprendizagem baseados na Web (ADL, 2004).

Um dos grandes diferenciais para a utilização do SCORM no desenvolvimento de conteúdo para Educação à Distância é seu foco na reusabilidade, acessibilidade, interoperabilidade e durabilidade. O SCORM tem como um de seus objetivos propiciar a independência de plataforma na qual os objetos serão utilizados, assim como facilitar a migração de cursos entre diferentes LMS que sejam compatíveis com esse modelo. A migração de um curso através de um processo de empacotamento ${ }^{2}$ conforme as especificações do SCORM demanda um esforço reduzido.

Além disso, o conteúdo desenvolvido em conformidade com SCORM é independente de contexto, ou seja, funcionará em situações variadas, seja inserido em um ambiente de gerenciamento de aprendizagem ou como parte de um curso on-line publicado diretamente na $W e b$ ou ainda em cenário híbrido.

Para a versão atual, SCORM 2004, a ADL publicou as especificações em quatro livros: Visão Geral (The SCORM Overview), Modelo de Agregação de Conteúdo (The SCORM Content Aggregation Model), Ambiente de Execução (The SCORM Runtime Environment) e Seqüênciamento e Navegação (The SCORM Sequencing \& Navigation). A figura 1 ilustra a organização do SCORM como um conjunto de especificações de outras organizações contidas ou referenciadas no modelo.

\footnotetext{
${ }_{1}^{1}$ LMS - Learning Management Systems - Sistemas de Gerenciamento de Aprendizagem

2 Empacotamento de conteúdo é um processo em que o designer instrucional agrega todos os objetos de aprendizagem de uma determinada unidade de aprendizagem em um único local, gerando um arquivo compactado denominado de pacote. 


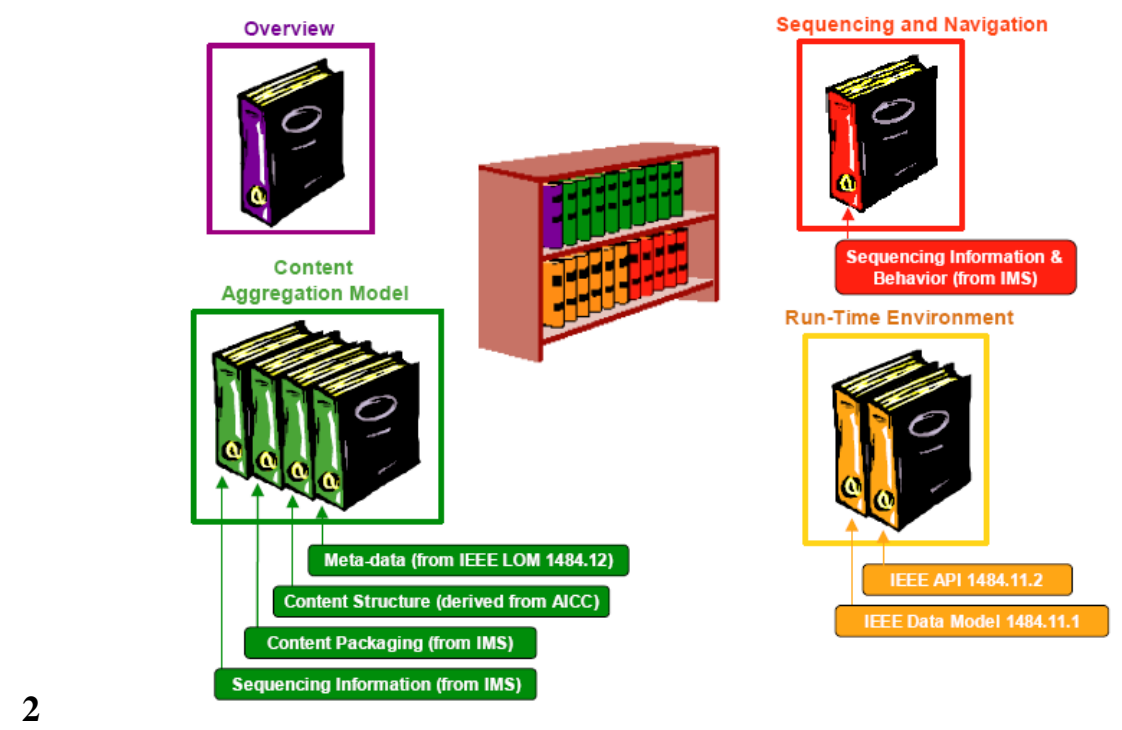

Figura 1 - SCORM como conjunto de especificações

No Modelo de Agregação de Conteúdo são definidos o Dicionário de Metadados, o Empacotamento de Conteúdos, a Estrutura de Conteúdos e o XML dos metadados do pacote. O Empacotamento de Conteúdo, em conjunto com a Estrutura de Conteúdos e o XML dos metadados do pacote, referem-se ao agrupamento, organização e identificação de todos os objetos de aprendizagem necessários para disponibilizar unidades de aprendizagem em diferentes LMS.

O SCORM também especifica os métodos para conduzir as comunicações entre o curso e o LMS. Isso é descrito como o Ambiente de Execução do SCORM, que inclui comunicações sobre a situação do curso, ou seja, quais materiais estão sendo apresentados para o estudante, assim como informações sobre o progresso do aluno durante o curso. A padronização dessas comunicações minimiza os problemas associados com a migração de cursos entre LMS diferentes, uma vez que tradicionalmente cada ambiente utiliza sua própria forma de rastreamento e gravação do progresso do aluno durante um curso.

A partir da versão 2004 (anteriormente denominada de 1.3) foi incorporado o livro de Seqüenciamento e Navegação advindo da $\mathrm{IMS}^{3}$. O Seqüenciamento e Navegação descreve como os conteúdos SCORM podem ser seqüenciados e como um LMS compatível deve interpretar regras de seqüenciamento.

No SCORM, o LMS é responsável pelo controle da distribuição dos objetos de aprendizagem aos estudantes obedecendo o que foi estabelecido na Agregação de Conteúdos e no Seqüenciamento e Navegação. O LMS tem a habilidade de determinar o que e quando deve ser entregue e rastrear o progresso do estudante durante o curso.

Como pôde ser observado, na sua versão atual, o SCORM se preocupa basicamente com o conteúdo, em como ele é organizado e seqüenciado, em como será mostrado e como rastrear as ações do aluno no que se refere à interação do conteúdo. Falta, porém, uma maneira de especificar como este "pacote" de conteúdos pode ser incorporado em

\footnotetext{
${ }^{3}$ IMS - IMS Learning Global Consortium, é uma organização sem fins lucrativos que visa sugerir padrões e modelos para a indústria de aprendizagem 
contextos que visem outras atividades de interação do aluno, além da interação autônoma entre o aluno e os objetos de aprendizagem.

\subsection{Os Objetos de Aprendizagem no contexto de sua aplicação em atividades de Ensino-Aprendizagem}

A discussão que muitas vezes se observa, é qual a melhor maneira do professor utilizar a tecnologia de Objetos de Aprendizagem. Olhando pelo ponto de vista do SCORM, observa-se um enfoque dos Objetos de Aprendizagem visando a automatização do processo de ensino-aprendizagem através de um LMS, minimizando a importância do professor. Entretanto, o que se percebe na vida real, é que os Objetos de Aprendizagem são uma excelente alternativa para apoiar as atividades do professor.

Voltando um pouco à sua origem, pode-se notar que a idéia de objetos de aprendizagem surgiu inicialmente pela grande preocupação na padronização do desenvolvimento e visualização de conteúdos visando seu reuso. Esta preocupação inicial adveio de um contexto no qual havia uma grande diversidade e formas de conteúdos. Adicionalmente, muitos destes cursos se apoiavam em uma grande autonomia do aluno onde a principal interação existente era entre o aluno e os conteúdos, ou seja, entre o aluno e os objetos de aprendizagem. Passado este esforço inicial, os professores e "projetistas" se deram conta de que para uma boa utilização dos Objetos de Aprendizagem, deve-se pensar em cursos nos quais a ênfase da interação vai além do contato do aluno com o material educacional. Deve-se pensar em cursos ou unidades de aprendizagem em que os Objetos de Aprendizagem se insiram em um contexto de interação mais amplo, levando-se também em consideração interações entre professores e alunos, bem como entre os próprios alunos.

Observando a utilização do SCORM em unidades de aprendizagem que são planejadas para ter outras atividades além da simples navegação entre os objetos de aprendizagem, verifica-se que todas as atividades de interação do curso, tais como fóruns e bate-papos, ocorrem em paralelo à exploração dos objetos de aprendizagem, dificultando a sincronização entre as atividades e os objetos de aprendizagem.

Outro problema é a existência várias abordagens pedagógicas normalmente utilizadas tanto no educação presencial como no educação a distância, tais como Projetos de Aprendizagem $^{4}$ e Aprendizagem Baseada em Problemas (PBL) ${ }^{5}$, entre outras, que são difíceis de especificar através do SCORM. Isto porque o SCORM não permite especificar o contexto e a abordagem de sua utilização e não aborda outros serviços comuns com a utilização de LMS, tais como sessões de fórum, sessões bate-papo, atividades desenvolvidas pelos alunos individualmente ou em grupo, etc.

Neste sentido, surgiram outras iniciativas para buscar uma maior abrangência nas pesquisas referentes ao desenvolvimento de Objetos de Aprendizagem. Uma dessas iniciativas tem ganhado gradativamente destaque, principalmente por seu arcabouço conceitual, o IMS Learning Design.

\footnotetext{
${ }^{4}$ Projetos de Aprendizagem - Abordagem pedagógica que se baseia na organização da prática pedagógica através de projetos elaborados a partir do interesse de alunos e professores e que exigem cooperação, pesquisa, curiosidade e desejo. Em seu desenvolvimento não há linearidade, pois impera o imprevisível. Essa abordagem tem como seus principais autores Hernandez e Ventura (1998).

${ }^{5}$ Problem Based Learning (Aprendizagem Baseada em Problemas) é uma abordagem pedagógica baseada em problemas e na sua investigação, de forma interativa em pequenos grupos independentes, nos quais os alunos integram-se e colaboram uns com os outros. Essa abordagem tem como um de seus principais autores Barrows (1985).
} 


\subsection{Learning Design}

O Learning Design da IMS, ou IMS Learning Design, é um modelo para especificação de objetos e atividades de aprendizagem baseada no EML (Educational Modeling Language) da Universidade Aberta da Holanda (OUNL - Open Universiteit Nederland). O desenvolvimento do EML iniciou-se em 1998 com a idéia de criar uma modelagem que pudesse representar uma unidade de aprendizagem em sua totalidade, englobando não só o conteúdo como também os diversos processos envolvidos (KOPER, 2002).

O IMS Learning Design dá suporte ao uso de diferentes abordagens de ensinoaprendizagem, tais como: behavioristas, cognitivistas e construtivistas. Isso é possível através de uma linguagem genérica e flexível, projetada para abranger diversos tipos de abordagens pedagógicas com a mesma tecnologia. O modelo descreve "Unidades de Aprendizagem", unidades elementares que provêem eventos de aprendizagem para aprendizes, satisfazendo um ou mais objetivos de aprendizagem (TATTERSAL e KOPER, 2003).

O IMS Learning Design (IMS, 2004), antigo EML, foi originalmente criado após um ampla avaliação e comparação entre as diversas abordagens pedagógicas e suas atividades de aprendizagem, buscando alcançar um meio termos entre aplicação pedagógica e um bom nível generalização.

O IMS Learning Design se diferencia principalmente do SCORM, que é mais voltado para o conteúdo, pois foi baseado na idéia de que existem mais relações no processo de ensino-aprendizagem, do que somente a relação de um único aluno diretamente com o conteúdo. Ele parte do princípio de que no processo de ensino-aprendizagem, existem além desta relação citada, a relação do aluno com o seu grupo de colegas, a relação do aluno com seu professor, a relação do aluno com as pessoas que dão suporte ao curso e também a relação do aluno com os recursos de aprendizagem (não somente o conteúdo, mas também as ferramentas e os objetos do mundo real).

Para o IMS Learning Design (LD), o processo de ensino-aprendizagem existe quando existem atividades de aprendizagem feitas pelos alunos com objetivos de aprendizagem definidos. Nesta visão ele surge como um framework para a descrição deste processo de uma forma geral, baseado principalmente no que se convencionou chamar de "Unidade de Aprendizagem".

Especificando um curso ou unidade de aprendizagem no IMS Learning Design, tem-se como resultado um documento XML que pode ser processado por uma aplicação "player", que coordena as interações dos estudantes e dos professores entre si e com os materiais educativos através da Web.

Historicamente a grande desvantagem do IMS Learning Design sob o SCORM sempre foi a ausência de ambientes de aprendizagem e softwares de autoria que dêem suporte a esta especificação. Entretanto em 2004 a OUNL desenvolveu um Runtime Engine, chamado CopperCore, que pode servir de interface entre o ambiente de aprendizagem e as unidades LD.

Baseado no CopperCore, foi desenvolvido pela University of Bolton o Reload Learning Design Player. Atualmente em versão beta o LD Player permite uma fácil importação de pacotes IMS Learning Design sem o uso de linhas de comando, automaticamente lendo e populando o LD player com um usuário ativo em cada Papel encontrado no arquivo de manifesto do IMS Learning Design. 
A ferramenta LD Editor disponibiliza uma interface de usuário intuitiva e simples para a edição de projetos baseados no IMS Learning Design. Possui um gerenciador de projetos para organizar e visualizar seus projetos LD e permite a visualização e edição de arquivos dentro da ferramenta. Adicionalmente o LD Editor disponibiliza assistentes para ajudar na importação e exportação de pacotes compactados do IMS Learning Design.

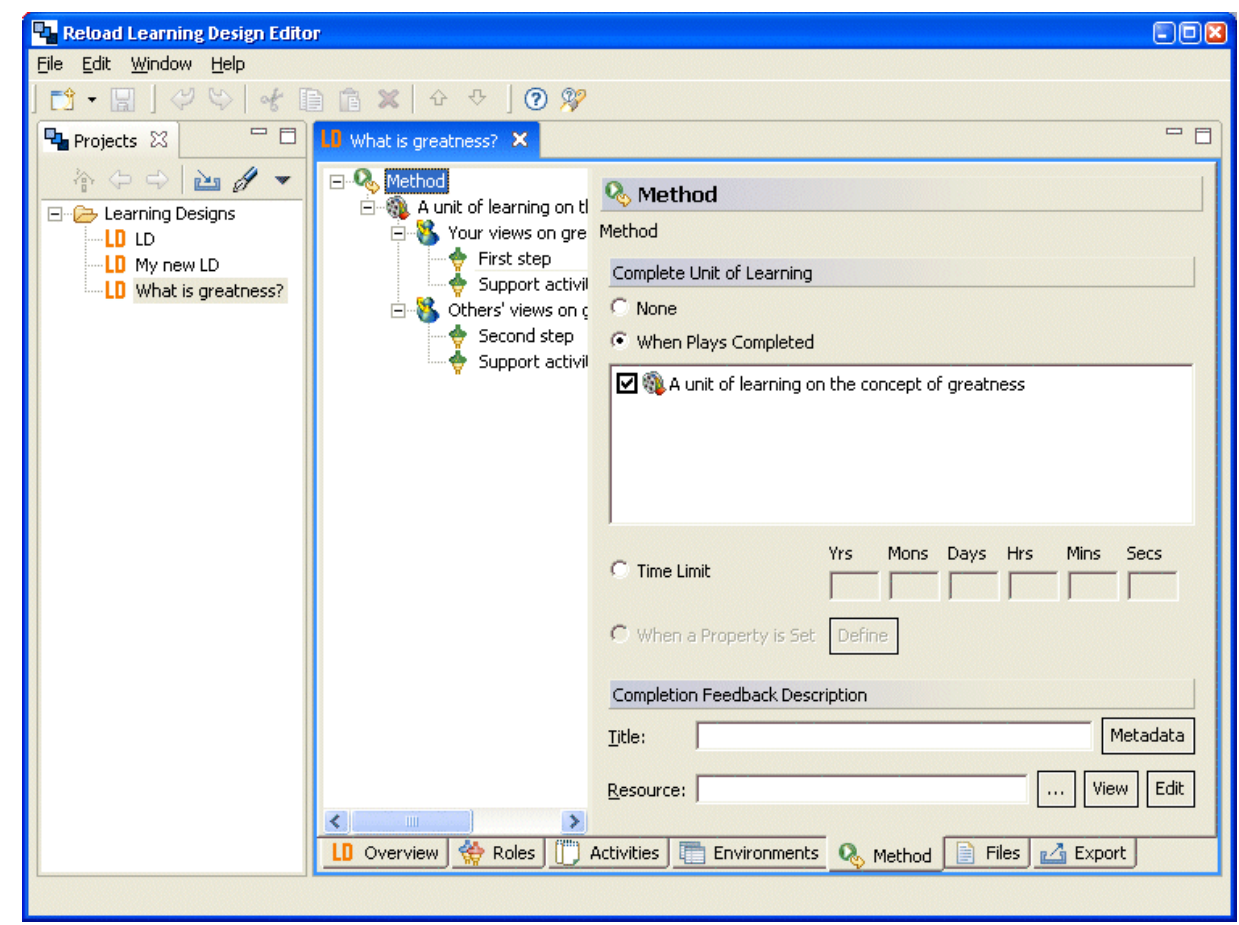

Figura 1 - Reload Learning Design Editor

Apesar de ainda em estágio inicial essas duas aplicações em conjunto com o Engine CopperCore, vêm complementar o que era considerado o grande ponto fraco desta abordagem, proporcionando aos professores e "projetistas" de unidades de aprendizagem, uma ferramenta poderosa e flexível. Mesmo assim, o IMS Learning Design ainda está longe de ter todo o suporte e aceitação de outras especificações mais consolidadas como o ADL SCORM.

\section{SCORM x Learning Design}

As duas especificações contêm características semelhantes ou complementares, pois ambas se utilizam padrões internacionais, tais como a própria IMS e a IEEE LTSC. As duas fazem partes de consócios que buscam a normalização e compatibilidade de soluções. A IMS que especificou o Learning Design é parte ativa da ADL que mantém o SCORM e as especificações de empacotamento e seqüenciamento do SCORM são da IMS. Ambas especificações utilizam-se da linguagem XML para diversos fins, desde do empacotamento das unidades de aprendizagem até a utilização de metadados. No quadro 1 pode-se verificar as principais diferenças entre as duas abordagens.

A grande vantagem do SCORM é sua crescente adoção como especificação de objetos de aprendizagem. Diversas ferramentas de autoria e LMS oferecem suporte ao SCORM. Isto parece ser resultado do grande esforço da ADL e do Departamento de Defesa norte- 
americano para consolidá-lo como modelo padrão. Outro fator provável é sua abrangência menor, focando mais no conteúdo do que no processo como um todo.

Analisando do ponto de vista das abordagens de aprendizagem, o SCORM, por hora, segue uma linha mais behaviorista, com seqüências de conteúdos pré-definidos, testes automáticos e critérios bastante objetivos. Isto inviabiliza utilização por diferentes abordagens ou metodologias.

Construir unidades de aprendizagem com enfoque construtivista, fica bastante difícil devido ao enfoque linear do SCORM, entretanto isto não impede de que apesar de linear os objetos individualmente possuam interação que de certa maneira possam promover naquele objeto uma aprendizagem construtivista.

\begin{tabular}{|c|c|c|}
\hline 3 & ADL SCORM 2004 & IMS Learning Design \\
\hline LMS com suporte & $\begin{array}{l}7 \text { WebCT, LearningSpace, } \\
\text { Moodle, Atutor, Aulanet, } \\
\text { WebAula, } \\
\text { LearningWise, Ilias, Alumni } \\
\text { Gestum, etc. }\end{array}$ & $\begin{array}{llr}8 & \text { Moodle } & (\mathrm{em} \\
\text { implementação) e } & \text { outros } \\
\text { baseados na } & \text { Engine } \\
\text { CooperCore. Reload } & \text { Player } \\
\text { (permite visualizar mas não é } \\
\text { LMS) }\end{array}$ \\
\hline $\begin{array}{lr}9 & \text { Ferramentas } \\
\text { Autoria compatíveis }\end{array}$ & $\begin{array}{l}\text { 10 Authorware, Flash MX, } \\
\text { Viewletbuilder, Toolbook, } \\
\text { Reload Editor, etc. LMSs com } \\
\text { funcionalidade para exportar em } \\
\text { SCORM }\end{array}$ & $\begin{array}{l}11 \text { Reload Editor que } \\
\text { permite criar pacotes LD a } \\
\text { partir de objetos de diversas } \\
\text { fontes }\end{array}$ \\
\hline $\begin{array}{lr}12 & \text { Flexibilidade para } \\
\text { diferentes } & \text { abordagens } \\
\text { pedagógicas } & \end{array}$ & $\begin{array}{l}13 \text { Projetado principalmente } \\
\text { para self-learning com conteúdos } \\
\text { mais seqüenciais e com pouca } \\
\text { flexibilidade }\end{array}$ & $\begin{array}{l}14 \text { Suporta as mais } \\
\text { variadas abordagens, bastando } \\
\text { combinar os objetos, os } \\
\text { serviços, os atores e seus } \\
\text { papéis nas atividades de } \\
\text { aprendizagem }\end{array}$ \\
\hline $\begin{array}{l}15 \text { Reusabilidade do } \\
\text { Conteúdo }\end{array}$ & $\begin{array}{l}16 \text { Conteúdo totalmente } \\
\text { reutilizável pelo mesmo LMS ou } \\
\text { por outros compatíveis com } \\
\text { SCORM }\end{array}$ & $\begin{array}{l}17 \text { Conteúdo totalmente } \\
\text { reutilizável pelo mesmo LMS } \\
\text { ou por outros compatíveis com } \\
\text { o LD }\end{array}$ \\
\hline $\begin{array}{ll}18 & \text { Interatividade } \\
\text { entre o } & \text { aluno e o conteúdo }\end{array}$ & $\begin{array}{l}19 \text { Permite, dependendo } \\
\text { mais da forma como o conteúdo } \\
\text { foi projetado }\end{array}$ & $\begin{array}{l}20 \text { Permite, dependendo } \\
\text { mais da forma como o } \\
\text { conteúdo foi projetado }\end{array}$ \\
\hline $\begin{array}{l}21 \text { Interação entre } \\
\text { aluno com professor e } \\
\text { outros alunos }\end{array}$ & Não suportado & $\begin{array}{l}23 \text { Prevê os papeis dos } \\
\text { participantes e o nível de } \\
\text { interação }\end{array}$ \\
\hline $\begin{array}{l}24 \text { Relação } r \text { entre } \\
\text { conteúdo e outras } \\
\text { ferramentas do LMS }\end{array}$ & Não suportado & $\begin{array}{l}26 \text { As atividades de } \\
\text { aprendizagem englobam os } \\
\text { objetos e os serviços do LMS }\end{array}$ \\
\hline $\begin{array}{ll}27 & \text { Entidade } \\
\text { Responsável }\end{array}$ & $\begin{array}{lcc}28 & \text { ADL } & - \\
\text { Distributed Learning }\end{array}$ & $\begin{array}{|lc|}29 & \text { IMS } \quad- \\
\text { Learning Consortium }\end{array}$ \\
\hline
\end{tabular}

Quadro 1 - Compativo entre o SCORM e o Learning Design 
Já o Learning Design permite outro tipo de reusabilidade, além da reusabilidade dos objetos, a reusabilidade de atividades de aprendizagem em outros contextos. O modelo especificado pelo Learning Design modela todo processo da unidade de aprendizagem, inclusive a abordagem pedagógica, e justamente por enxergar o processo como um todo, proporcionará a construção de unidades de aprendizagem mais consistentes e coerentes.

Sua maior desvantagem é justamente a ausência de um grande leque de LMS e softwares de autoria que dêem suporte a esta especificação.

\section{Conclusões}

Quando se pensa em modelos de utilização de Objetos de Aprendizagem para dar suporte às atividades do professor, logo nos vem a cabeça o popular modelo SCORM, mesmo sendo este um modelo pensado para uma abordagem em que o professor participa em sua maior parte somente na fase de planejamento.

A utilização dos Objetos de Aprendizagem pode ser vista em um contexto maior do que simplesmente uma sequiência de conteúdos. Do ponto de vista do professor, levando-se em consideração tanto em atividades a distância como atividades presenciais e também do ponto de vista do processo como um todo, verifica-se que o IMS Learning Design, uma vez mais popularizado, pode se tornar uma importante ferramenta, tanto para dar suporte ao processo de ensino-aprendizagem, como também no processo de planejamento.

Neste sentido, o SCORM apesar de ser muito mais difundido e utilizado, no momento, ainda é menos abrangente, podendo com o tempo ceder espaço ao IMS Learning Design, ainda pouco adotado, mas com muito mais flexibilidade e abrangência.

\section{Referências:}

Advanced Distributed Learning (ADL). Disponível em: http://www.adlnet.org. Acesso em: 13 mar. 2005.

Advanced Distributed Learning. Sharable Content Object Reference Model - SCORM $20042^{\text {nd }}$ Edition - Overview. Julho, 2004.

BARROWS, Howard S. How to design a problem-based curriculum for the preclinical years. New York: Springer, 1985.

HERNÁNDEZ, Fernando; VENTURA, Montserrat. A organização do currículo por projetos de trabalho: O conhecimento é um caleidoscópio. Porto Alegre: Artes Médicas, 1998.

IMS Global Learning Consortium Inc. Learning Design Specification Disponível em: http://www.imsglobal.org/learningdesign/index.html. Acesso em: 05 abr.2005.

KOPER, R., Educational Modelling Language :adding instructional design to existing specifications. 2002, Open University of the Netherlands.

TAROUCO, L. M. R.; FABRE M. J. M.; TAMUSIUNAS, F. R.. Reusabilidade de objetos educacionais. RENOTE - Revista Novas Tecnologias na Educação: II Ciclo de Palestras sobre Novas Tecnologias na Educação. Porto Alegre, RS, 2003 